\title{
Effect of artificial aging at QUV on the color of finish treated woods of Pinus sylvestris and Quercus robur
}

\section{Effet du vieillissement artificiel au QUV sur la couleur des bois de Pinus sylvestris et Quercus robur traités par finition}

\author{
A. Hakam ${ }^{1}$, N. El Imame ${ }^{1,2}$, M. El kortbi ${ }^{1}$, M. El Matar ${ }^{2}$, M. Ziani ${ }^{1}$ and A. Merlin ${ }^{3}$ \\ ${ }^{1}$ Wood Science Laboratory, Mohammed V University in Rabat, Faculty of Science, PO Box 1014, Morocco \\ ${ }^{2}$ Centre Technique des Industries du Bois et de l'Ameublement, Sidi Maârouf, Casablanca, Maroc \\ ${ }^{3}$ Université de Lorraine, Faculté des Sciences et Technologies, BP 70239 - 54506 Vandoeuvre Les Nancy Cedex, France
}

\begin{abstract}
The durability of exterior finishes on wood is not assured with the systems currently used. Thus, the objective of this study was to evaluate the color change of the finishes applied to wood, the most commercialized in Morocco, in order to find the wood-finishing couple that has the best durability in outdoor use. QUV accelerated aging tests were carried out during 2016 hours, according to the standard (NF 927-6 2006), on Scots pine (Pinus sylvestris) and Pedunculate oak (Quercus robur) coated with four finishes (two polyurethane finishes $F_{1}$ and $F_{2}$ and two acrylic finishes $F_{3}$ and $\left.F_{4}\right)$. Colorimetric measurements in color system CIELab: Gloss $\left(\mathrm{L}^{*}\right)$, green-red $\left(\mathrm{a}^{*}\right)$ and blue-yellow $\left(\mathrm{b}^{*}\right)$ coordinates, color saturation $\left(\mathrm{C}^{*}\right)$ and tone angle ( $\left.\mathrm{H}^{*}\right)$ were performed using an X-Rite Model SP62 spectrophotometer before and at the end of the QUV accelerated aging treatment. The results showed that QUV aging caused a decrease in the L* value and a change in the surface color of all the finishing pairs studied. For wood / finishing couples with Quercus robur as a wooden support, the couple Quercus robur / F3 has the best resistance to color change, while for couples wood / finish with Pinus sylvestris as a wooden support, the couple Pinus sylvestris / F1 has the best resistance to color change.
\end{abstract}

Key words: Wood, finish, gloss, color, accelerated aging, QUV.

Résumé. La durabilité des finitions extérieures sur le bois n'est pas assurée avec les systèmes employés actuellement. Ainsi, l'objectif de cette étude était d'évaluer le changement de couleur des finitions appliquées aux bois, les plus commercialisés au Maroc, dans le but de trouver le couple bois-finition qui présente la meilleure durabilité en utilisation extérieure. Des tests de vieillissement accéléré au QUV ont été réalisés pendant 2016 heures, selon la norme (NF 927-6 2006), sur le pin sylvestre (Pinus sylvestris) et le chêne pédonculé (Quercus robur) revêtus de quatre finitions (deux finitions polyuréthanes F1 et F2 et deux finitions acryliques F3 et F4). Les mesures colorimétriques dans le système de couleur CIELab: La brillance (L*), les coordonnées vert-rouge $\left(\mathrm{a}^{*}\right)$ et bleu-jaune $\left(\mathrm{b}^{*}\right)$, la saturation des couleurs $\left(\mathrm{C}^{*}\right)$ et l'angle de tonalité $\left(\mathrm{H}^{*}\right)$ ont été réalisées à l'aide d'un spectrophotomètre X-Rite Model SP62, avant et à la fin du traitement de vieillissement accéléré au QUV. Les résultats ont montré que le vieillissement au QUV a provoqué une diminution de la valeur de $\mathrm{L}^{*}$ et une modification de la couleur de surface de tous les couples de finition étudiés. Pour les couples bois/finition avec le Quercus robur comme support en bois, le couple Quercus robur / $\mathrm{F}_{3}$ présente la meilleure résistance au changement de couleur, alors que pour les couples bois / finition avec le Pinus sylvestris comme support en bois, le couple Pinus sylvestris / $\mathrm{F}_{1}$ présente la meilleure résistance au changement de couleur.

Mots clés : Bois, finition, brillance, couleur, vieillissement accéléré, QUV. 


\section{Introduction}

Les principaux facteurs de dégradation des composants en bois à l'extérieure sont l'exposition aux rayons ultraviolets et à l'eau [1-5].

Pour la protection de l'aspect naturel du bois et sa couleur contre les intempéries, des recherches sur les revêtements transparents ou semi-transparents, tels que des vernis, des lasures ou des peintures ont été réalisées [6-9].

Le test de vieillissement au QUV consiste à évaluer la résistance au vieillissement accéléré au QUV du système de revêtement appliqué sur un support en bois. Ce test a l'avantage d'être accéléré par rapport au vieillissement naturel [10-11], qui dure un an et qui présente souvent des problèmes de répétabilité et de reproductibilité.

Le vieillissement artificiel ou accéléré influe sur la couleur du bois traité ou non traité par finition [12-19].

La variation de la couleur de finition observée varie selon la nature du produit de finition, de l'espèce de bois [20-22] et de son traitement, thermique [23-27] ou chimique [28].

D'autres études ont été consacrées à l'effet du vieillissement accéléré sur l'adhérence des finitions [29].

\section{Matériaux et méthodes}

\subsection{Matériaux}

Un système Bois / Finition est un échantillon qui est composé d'un support bois et un ou plusieurs produits de finition déposés sur ce bois.

Le choix des systèmes Bois/Finitions a été réalisé en étroite concertation avec les industriels de la filière boisfinition au Maroc.

Le choix du support bois s'est porté sur le Pin sylvestre (Pinus sylvestris) et le Chêne pédonculé (Quercus robur).

Le tableau 1 donne quelques caractéristiques du support bois utilisé

Tableau 1. Caractéristiques du support bois utilisé

\begin{tabular}{|c|c|c|}
\hline & (Pinus sylvestris) & (Quercus robur) \\
\hline Type de bois & résineux & feuillus \\
\hline Densité* & $\sim 0.55$ & $\sim 0.74$ \\
\hline Structure & Aucun vaisseau & Cernes poreux \\
\hline
\end{tabular}

\begin{tabular}{|l|c|c|}
\hline $\begin{array}{l}\text { Largeur de } \\
\text { cerne }(\mathrm{mm})\end{array}$ & $\sim 1,4$ & $\sim 1,4$ \\
\hline \multicolumn{3}{|c|}{ *Tropix 7 [30] }
\end{tabular}

\subsubsection{Confection des éprouvettes}

Des éprouvettes, de dimensions $15 \times 8 \times 1,8 \mathrm{~mm}$, ont été coupées en longueur suivant l'axe de symétrie longitudinal (L), en largeur suivant l'axe de symétrie tangentiel (T) et en profondeur suivant l'axe de symétrie radial (R). Toutes les éprouvettes en bois ont été équilibrées dans une chambre climatique à une humidité relative de $65 \pm 5 \%$ et à une température de $20{ }^{\circ} \mathrm{C}$ avant le revêtement par la finition. L'humidité d'équilibre des éprouvettes était de $12 \%$.

\subsubsection{Produit de finition}

Comme pour le bois, les quatre finitions aqueuses commerciales utilisées ont été choisies en concertation avec les industriels de la filière bois-finition au Maroc.

Deux finitions à base de résine polyuréthane $\left(\mathrm{F}_{1}\right.$ et $\left.\mathrm{F}_{2}\right)$ et deux à base de résine acrylique $\left(\mathrm{F}_{3}\right.$ et $\left.\mathrm{F}_{4}\right)$. Le tableau 2 donne quelques indications sur les finitions commerciales utilisées.

Tableau 2. Caractéristiques du support bois utilisé

\begin{tabular}{|c|c|c|}
\hline Finition & Résine de base & Type du produit \\
\hline $\mathrm{F}_{1}$ & Polyuréthane & Vernis aqueux transparent \\
\hline $\mathrm{F}_{2}$ & Polyuréthane & Vernis aqueux mate blanc \\
\hline $\mathrm{F}_{3}$ & Acrylique & $\begin{array}{l}\text { Lasure aqueuse } \\
\text { Transparente mate }\end{array}$ \\
\hline $\mathrm{F}_{4}$ & Acrylique & $\begin{array}{l}\text { Lasure Aqueuse opaque } \\
\text { blanche mate }\end{array}$ \\
\hline
\end{tabular}

\subsubsection{Revêtement des surfaces en bois}

Les produits de finition choisis ont été appliqués selon les fiches techniques de chaque produit sur une des faces tangentielles de chaque éprouvette. Les autres faces ont été fermées avec un produit scellant, appliqué pendant trois jours, à raison d'une couche par jour.

Avant le début des essais, les éprouvettes ont été laissées sécher pendant une semaine.

\subsection{Méthode}

2.2.1 Vieillissement accéléré au QUV 
L'expérience de vieillissement accéléré au QUV a été réalisée dans un QUV de QLAb avec huit lampes UVA 340 selon la norme EN 927-6 2006. Un cycle d'exposition des éprouvettes dans le QUV implique une première phase de condensation pendant $24 \mathrm{~h}$ à $\mathrm{T}=45 \pm$ $3^{\circ} \mathrm{C}$, suivie d'une phase d'irradiation à $1^{\prime} \mathrm{UV}$ et pulvérisation pendant $144 \mathrm{~h}$ à $\mathrm{T}=60 \pm 3^{\circ} \mathrm{C}$, puis une phase d'irradiation de $2,5 \mathrm{~h}$ et une dernière phase de pulvérisation pendant $0,5 \mathrm{~h}$. Les échantillons ont été exposés à l'altération accélérée pendant 12 semaines.

\subsubsection{Mesures de la couleur}

L'analyse colorimétrique des systèmes bois-finition a été effectuée en utilisant un spectrophotomètre X-rite SP 62.

Les paramètres mesurés sont : la brillance $\left(\mathrm{L}^{*}\right)$, les coordonnées chromatiques, vert-rouge $\left(\mathrm{a}^{*}\right)$ et bleu-jaune $\left(b^{*}\right)$ en référence au système de coordonnées de couleur CIELab. La valeur retenue pour chaque paramètre est la valeur moyenne de trois mesures.

A partir des valeurs de $L^{*}, a^{*}$, et $b^{*}$, la différence de brillance $\left(\Delta \mathrm{L}^{*}\right)$ et de coordonnées chromatiques $\left(\Delta \mathrm{a}^{*}\right.$ et $\left.\Delta \mathrm{b}^{*}\right)$, l'angle de teinte (h), la saturation de couleur $\left(\mathrm{C}^{*}\right)$ et la différence globale de couleur $\left(\Delta \mathrm{E}^{*}\right)$ ont été calculés en utilisant les formules suivante :

$$
\begin{aligned}
& \Delta \mathrm{L}^{*}=\mathrm{L}^{*}{ }_{\mathrm{f}}-\mathrm{L}^{*}{ }_{\mathrm{i}} \\
& \Delta \mathrm{a}^{*}=\mathrm{a}^{*}{ }_{\mathrm{f}}-\mathrm{a}^{*} \mathrm{i}_{\mathrm{i}} \\
& \Delta \mathrm{b}^{*}=\mathrm{b}^{*} \mathrm{f}-\mathrm{b}^{*} \mathrm{i} \\
& \mathrm{h}=\operatorname{arctg}\left(\mathrm{b}^{*} / \mathrm{a}^{*}\right) \\
& \mathrm{C}^{*}=\left(\mathrm{a}^{* 2}+\mathrm{b}^{* 2}\right)^{1 / 2} \\
& \Delta \mathrm{E}^{*}=\left(\Delta \mathrm{L}^{* 2}+\Delta \mathrm{a}^{* 2}+\Delta \mathrm{b}^{* 2}\right)^{1 / 2}
\end{aligned}
$$

où, $L^{*} a^{*}{ }_{f} b^{*}{ }_{f}$ sont $L^{*}$, a* et $b^{*}$ des spécimens après essai au QUV et $L^{*}, a^{*}{ }_{i}$ et $b^{*}{ }_{i}$ sont $L^{*}, a^{*}$ et $b^{*}$ des échantillons avant essai au QUV, respectivement.

\section{Résultats et discussion}

Le tableau 3 présente les valeurs de $L^{*}, a^{*}, b^{*}$ et $E^{*}$ basées sur les coordonnées de couleur initiales pour chaque couple bois / finition, le tableau 4 présente les valeurs de $\Delta \mathrm{L}^{*}, \Delta \mathrm{a}^{*}, \Delta \mathrm{b}^{*}, \Delta \mathrm{E}^{*}, \mathrm{C}^{*}$ et $\mathrm{H}$, calculées à partir des valeurs de $L^{*}, a^{*}, b^{*}$ et $E^{*}$ basées sur les coordonnées de couleur initiales pour chaque couple bois / finition et le tableau 5 présente quelques photos des échantillons avant et après vieillissement au QUV.

Comme on peut le voir dans les tableaux 3 et 4 , les valeurs de $L^{*}$ de tous les couples de finition diminuent après vieillissement au QUV, mais avec un impact plus important sur les couples (Pinus sylvestris / Finition), tandis que les valeurs $\mathrm{a}^{*}$ augmentent.

Les valeurs positives de $\Delta \mathrm{a}^{*}$ montrent une tendance de la surface de la finition à devenir plus rougeâtre, sauf pour le couple (Quercus robur / F2), où la surface à tendance à devenir plus verdâtre. La coordonnée vert- rouge $\left(\mathrm{a}^{*}\right)$ des couples (Pinus sylvestris / Finition) était plus élevée que celle des couples (Quercus robur / Finition) et la coordonnée bleu-jaune ( $\left.\mathrm{b}^{*}\right)$ augmente pour les couples avec finition $F_{1}$ et le couple (Quercus robur / $\mathrm{F}_{4}$ ) et diminue pour les couples avec finition $\mathrm{F}_{2}$ et $\mathrm{F}_{3}$ et le couple (Pinus sylvestris / $\mathrm{F}_{4}$ ).

Les couples (Pinus sylvestris / finition) présentaient des valeurs plus élevées de saturation des couleurs $\left(\mathrm{C}^{*}\right)$ que les couples (Quercus robur / Finition). Comme on peut le constater dans le tableau 4, les valeurs de la saturation de couleur $\left(\mathrm{C}^{*}\right)$ des couples avec les finitions $F_{1}$ et $F_{4}$ augmentent, tandis qu'elles diminuent pour les couples avec les finitions $F_{2}$ et $F_{3}$, après vieillissement au QUV. La valeur $\mathrm{C}^{*}$ résulte des coordonnées $\mathrm{a}^{*}$ et $\mathrm{b}^{*}$, et les valeurs les plus élevées de $\left(b^{*}\right)$ sont ceux des couples (Pinus sylvestris / finition), ce que signifie que la saturation des couleurs $\left(\mathrm{C}^{*}\right)$ suit les valeurs de $\left(\mathrm{b}^{*}\right)$ des couples (Pinus sylvestris / finition). Le tableau 4 montre également que l'angle de tonalité $\left(\mathrm{H}^{*}\right)$ diminue pour tous les couples de finition après vieillissement au QUV. D'après les résultats du tableau 4 , le classement de la durabilité des systèmes Quercus robur/Finition par ordre décroissant est : Quercus robur / $\mathrm{F}_{3}$, Quercus robur / $\mathrm{F}_{4}$, Quercus robur / $\mathrm{F}_{1}$ et Quercus robur / $\mathrm{F}_{2}$ et le classement de la durabilité des systèmes Pinus sylvestris / Finition par ordre décroissant est: Pinus sylvestris / $\mathrm{F}_{1}$, Pinus sylvestris / $\mathrm{F}_{4}$, Pinus sylvestris / $\mathrm{F}_{2}$ et Pinus sylvestris / $\mathrm{F}_{3}$.

\section{Conclusion}

La finition protège le bois contre l'humidité, la température et l'ensoleillement. C'est une étape importante pour valoriser les produits en bois. D'ailleurs, la durée de vie de ces derniers est souvent associée à la durabilité de son revêtement de finition. Dans cette étude, l'effet de l'altération accélérée sur la brillance et la couleur des spécimens de pin sylvestre (Pinus sylvestris) et de Chêne pédonculé (Quercus robur) revêtus de vernis et de lasure a été étudié en effectuant des tests de la brillance et de la couleur de surface. Le choix des systèmes Bois/Finitions a été réalisé en concertation avec les industriels de la filière bois-finition au Maroc. Deux finitions à base de résine polyuréthane $\left(\mathrm{F}_{1}\right.$ et $\left.\mathrm{F}_{2}\right)$ et deux à base de résine acrylique $\left(\mathrm{F}_{3}\right.$ et $\left.\mathrm{F}_{4}\right)$ ont été choisies. Les résultats ont montré que l'altération provoquait une diminution de la brillance et une modification de la couleur de tous les spécimens étudiés. Cette étude a permis de faire un classement de la durabilité des systèmes bois / finition étudiés.

Le classement de la durabilité des systèmes Quercus robur/Finition par ordre décroissant est : Quercus robur / $\mathrm{F}_{3}$, Quercus robur / $\mathrm{F}_{4}$, Quercus robur / $\mathrm{F}_{1}$ et Quercus robur / $\mathrm{F}_{2}$ et le classement de la durabilité des systèmes Pinus sylvestris / Finition par ordre décroissant est: 
Pinus sylvestris / $\mathrm{F}_{1}$, Pinus sylvestris / $\mathrm{F}_{4}$, Pinus sylvestris $\quad / \mathrm{F}_{2}$ et Pinus sylvestris / $\mathrm{F}_{3}$.

Tableau 3. Résultats des mesures de la couleur

\begin{tabular}{|c|c|c|c|c|c|c|c|c|c|}
\hline \multirow{2}{*}{ Finition } & \multirow{2}{*}{ Bois } & \multicolumn{2}{|c|}{$\mathrm{L}^{*}$} & \multicolumn{2}{|c|}{$\mathrm{a}^{*}$} & \multicolumn{3}{c|}{$\mathrm{b}^{*}$} & \multicolumn{3}{c|}{$\mathrm{E}^{*}$} \\
\cline { 3 - 10 } & & Avant & Après & Avant & Après & Avant & Après & Avant & Après \\
\hline \multirow{3}{*}{ F1 } & $\mathrm{P}$ & 64,87 & 54,43 & 11,28 & 14,85 & 27,56 & 28,94 & 71,38 & 63,41 \\
\cline { 2 - 11 } & $\mathrm{C}$ & 58,98 & 51 & 7,49 & 10,16 & 22,78 & 24,1 & 63,67 & 57,32 \\
\hline \multirow{2}{*}{ F2 } & $\mathrm{P}$ & 66,12 & 51,08 & 11,17 & 13,8 & 30,45 & 22,89 & 73,65 & 57,65 \\
\cline { 2 - 11 } & $\mathrm{C}$ & 55,88 & 67,77 & 8,62 & 5,44 & 24,34 & 15,54 & 61,56 & 69,74 \\
\hline \multirow{3}{*}{ F3 } & $\mathrm{P}$ & 60,85 & 42,69 & 13,26 & 13,47 & 26,24 & 20,63 & 67,58 & 49,29 \\
\cline { 2 - 10 } & $\mathrm{C}$ & 53,3 & 48,51 & 10,77 & 11,61 & 26,76 & 24,07 & 60,61 & 55,38 \\
\hline \multirow{2}{*}{ F4 } & $\mathrm{P}$ & 65,45 & 48,49 & 11,88 & 17,32 & 22,67 & 21,23 & 70,28 & 55,70 \\
\cline { 2 - 10 } & $\mathrm{C}$ & 63,9 & 55,09 & 7,18 & 13,84 & 21,64 & 27,81 & 67,85 & 63,24 \\
\hline
\end{tabular}

$\mathrm{P}$ : Pinus sylvestris et $\mathrm{C}:$ Quercus robur

Tableau 4. Résultats des mesures de la couleur

\begin{tabular}{|c|c|c|c|c|c|c|c|c|c|}
\hline \multirow[b]{2}{*}{ Finition } & \multirow[b]{2}{*}{ Bois } & \multirow[b]{2}{*}{$\Delta \mathrm{L}^{*}$} & \multirow[b]{2}{*}{$\Delta \mathrm{a}^{*}$} & \multirow[b]{2}{*}{$\Delta \mathrm{b}^{*}$} & \multirow[b]{2}{*}{$\Delta \mathrm{E}^{*}$} & \multicolumn{2}{|c|}{$\mathrm{C}^{*}$} & \multicolumn{2}{|c|}{$\mathrm{H}^{*}$} \\
\hline & & & & & & Avant & Après & Avant & Après \\
\hline \multirow{2}{*}{ F1 } & $P$ & $-10,44$ & 3,57 & 1,38 & $-7,97$ & 29,78 & 32,53 & 1,18 & 1,1 \\
\hline & $\mathrm{C}$ & $-7,98$ & 2,67 & 1,32 & $-6,35$ & 23,98 & 26,15 & 1,25 & 1,17 \\
\hline \multirow{2}{*}{ F2 } & $P$ & $-15,04$ & 2,63 & $-7,56$ & $-16,00$ & 32,43 & 26,72 & 1,22 & 1,03 \\
\hline & $\mathrm{C}$ & 11,89 & $-3,18$ & $-8,8$ & 8,18 & 25,82 & 16,46 & 1,23 & 1,23 \\
\hline \multirow{2}{*}{ F3 } & $P$ & $-18,16$ & 0,21 & $-5,61$ & $-18,29$ & 29,4 & 24,64 & 1,1 & 0,99 \\
\hline & $\mathrm{C}$ & $-4,79$ & 0,84 & $-2,69$ & $-5,22$ & 28,84 & 26,73 & 1,19 & 1,12 \\
\hline \multirow{2}{*}{ F4 } & $\mathrm{P}$ & $-16,96$ & 5,44 & $-1,44$ & $-14,58$ & 25,59 & 27,4 & 1,09 & 0,89 \\
\hline & $\mathrm{C}$ & $-8,81$ & 6,66 & 6,17 & $-4,60$ & 22,8 & 31,06 & 1,25 & 1,11 \\
\hline
\end{tabular}

$\mathrm{P}$ : Pinus sylvestris et C : Quercus robur

Tableau 5. Photos des échantillons avant et après vieillissement au QUV

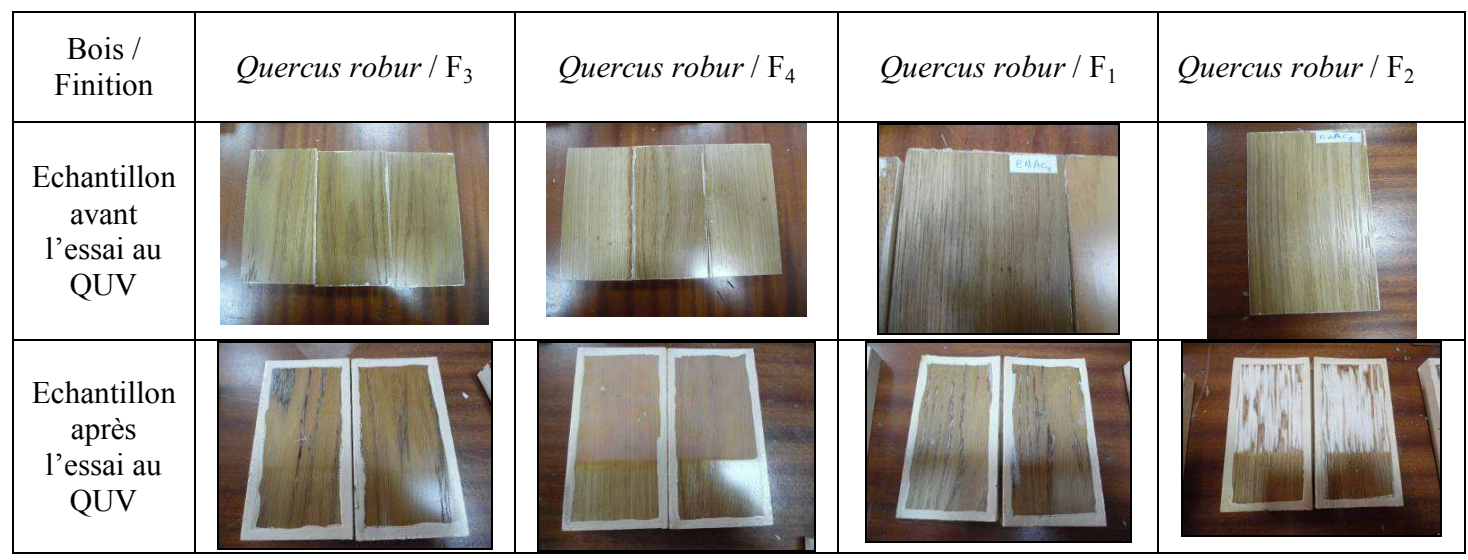




\begin{tabular}{|c|c|c|c|c|}
\hline $\begin{array}{c}\text { Bois / } \\
\text { Finition } \\
\end{array}$ & Pinus sylvestris/F1 & Pinus sylvestris/F4 & Pinus sylvestris/ F2 & Pinus sylvestris/F3 \\
\hline $\begin{array}{c}\text { Echantillon } \\
\text { avant } \\
\text { l'essai au } \\
\text { QUV }\end{array}$ & & & & \\
\hline $\begin{array}{c}\text { Echantillon } \\
\text { après } \\
\text { l'essai au } \\
\text { QUV }\end{array}$ & & & & \\
\hline
\end{tabular}

\section{Références}

1. W.C. Feist and D.N.S. Hon, Chemistry of Weathering and Protection (American Chemical Society, 1984)

2. M.K. Yalinkilic, R. Ilhan, Y. Imamura, M. Takahashi, Z. Demirci, A.C. Yalinkilic and H. Peker, J. Wood Sci. 45, 502 (1999)

3. J. Zhang and P.D. Kamdem, Weathering of copper-amine treated wood (IRG, Kona, Hawaii, 2000)

4. B. George, E. Suttie, A. Merlin and X. Deglise, Polymer Degradation and Stability 88, 268 (2005)

5. J. Zhang, P. Kamdem, A. Temiz, Applied Surface Science 256, 842 (2009)

6. V. Jirous-Rajkovic and H. Turkulin, Drvna Industrija 53, 9 (2002)

7. V. Jirous-Rajkovic, A. Bogner and D. Radovan, Surface Coat International Part B - Coat Trans 87, 15 (2004)

8. E. Scrinzi, S. Rossi, F. Deflorian and C. Zanella, Prog. Org. Coat. 72, 81 (2011)

9. G. Grüll, M. Truskaller, L. Podgorski, S. Bollmus and F. Tscherne, Eur. J. Wood Prod. 69, 443 (2011)

10. T. Turkoglu, E. Baysal, I. Kureli, H. Toker and M. E. Ergun, Wood research 60, 833 (2015)

11. P.R.Kerber, D.M. Stangerlin, E. Pariz, R.R. de Melo, A.P. de Souza and L. Calegari, Nativa, Sinop, 4, 303 (2016)

12. A. Temiz , U.C. Yildiz, I. Aydin, M. Eikenes, G. Alfredsen, G. Colakoglu, Applied Surface Science 250, 35 (2005)

13. S. Zahri, C. Belloncle, F. Charrier, P. Pardon, S. Quideau and B. Charrier, Applied Surface Science 253, 4985 (2007)

14. N. Çakıcıer, S. Korkut, D.S. Korkut, A. Kurtoğlu and A. Sönmez, International Journal of the Physical Sciences 6, 1929 (2011)

15. E. Baysal, E.D. Tomak, M. Ozbeyc and E. Altinc, Coloration Technology 130, 140 (2013)
16. R.F. Teles, A.F. da Costa. Colorimetric evaluation of three tropical woods after accelerated weathering (45th IRG, St George, Utah, USA, 2014)

17. T. Krystofiak, B. Lis, M. Muszyńska, S. Proszyk, Drewno, 59, 127 (2016)

18. E. Baysal, E.D. Tomak, E. Topaloglu, E. Pesman, Maderas. Ciencia y tecnología 18, 253 (2016)

19. M. Yalcin, H. Pelit, C. Akcay and N. Cakicier, Coloration Technology 133, 334 (2017)

20. N. Gierlinger, D. Jacques, M. Grabner, R. Wimmer, M. Schwanninger, P. Rozenberg and L.E. Pâques, Trees 18,102 (2004)

21. A.J.V. Zanuncio, J.P. Motta, T.A. DA Silveira, E.S. Farias and P.F. Trugilho, BioResources 9, 293 (2014)

22. A.J.V. Zanuncio, A.G. Carvalho, M.T. de Souza, C.M. Jardim, A. de C.O. Carneiro, J.L. Colodette, Maderas Ciencia y tecnología 17, 857 (2015)

23. N. Ayadi, F. Lejeune, F. Charrier, B. Charrier and A. Merlin, Holz als Roh- und Werkstoff 61, 221 (2003)

24. P; Bekhta and P. Niemz, Holzforschung 57, 539 (2003)

25. A.L. Piedade S.M. Pincelli, L.F. de Moura, J.O. Brito, Maderas. Ciencia y tecnología, 14, 239 (2012)

26. P.H.G. de Cademartori, E. Schneid, D.A. Gatto, D.M. Stangerlin and R. Beltrame. Maderas Ciencia y tecnología 15, 57 (2013)

27. Z. Demirci, A. Sönmez and M. Budakç, BioResources 8,1852 (2013)

28. E. Baysal, T. Turkoglu, E. Gavcar, H. Toker, A. Gunduz, M. Kucuktuvek, H. Peker and D. K. Bayraktar, Wood research 62, 737 (2017)

29. A. Sonmez, M. Budakci and M. Bayram, Scientific Research and Essay 4, 1432 (2009)

30. J. Gérard, D. Guibal, S. Paradis, M. Vernay, J. Beauchêne, L. Brancheriau and A. Thibaut, (Tropix 7 Version 7.5.1 CIRAD 2011). https://doi.org/10.18167/74726f706978 